\section{BRAZIULIAN JOURNAL}

OF MEDICAL AND BIOLOGICAL RESFARCH

www.bjournal.com.br
ISSN 0100-879X

Volume 43 (5) 381-496 May 2011

BIOMEDICAL SCIENCES

AND

CLINICAL INVESTIGATION

Braz J Med Biol Res, May 2011, Volume 44(5) 402-410

doi: 10.1590/S0100-879X2011007500041

Normal skin and hypertrophic scar fibroblasts differentially regulate collagen and fibronectin expression as well as mitochondrial membrane potential in response to basic fibroblast growth factor

Rui Song, Hui-Ning Bian, Wen Lai, Hua-De Chen and Ke-Seng Zhao

The Brazilian Journal of Medical and Biological Research is partially financed by

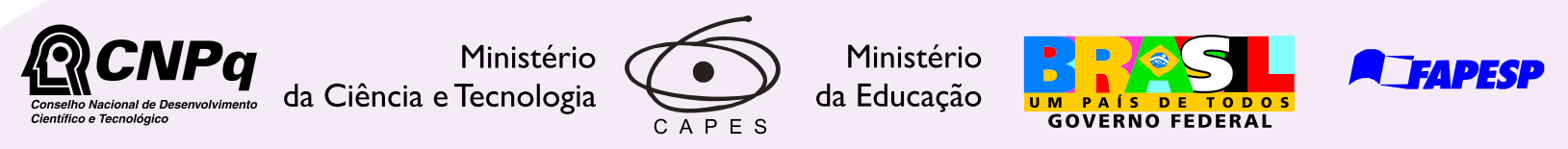

Institutional Sponsors

$\operatorname{sip} 0_{0}$
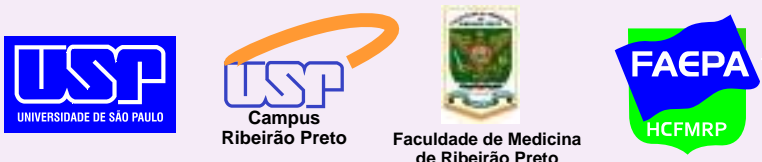

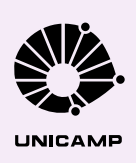

$\oplus$ SHIMADZU

GE Healthcare
Hotsite of proteomics metabolomics developped by:

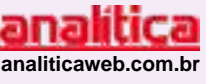

Thermo SCIENTIFIC 


\title{
Normal skin and hypertrophic scar fibroblasts differentially regulate collagen and fibronectin expression as well as mitochondrial membrane potential in response to basic fibroblast growth factor
}

\author{
Rui Song ${ }^{1}$, Hui-Ning Bian², Wen Lai ${ }^{2}$, Hua-De Chen ${ }^{2}$ and Ke-Seng Zhao ${ }^{1}$ \\ ${ }^{1}$ Guangdong Key Laboratory of the Shock and Microcirculation Research, Department of Pathophysiology, \\ Southern Medical University, Guangzhou, China \\ ${ }^{2}$ Department of Burns, Guangdong General Hospital, Guangdong Academy of Medical Sciences, \\ Guangzhou, China
}

\begin{abstract}
Basic fibroblast growth factor (bFGF) regulates skin wound healing; however, the underlying mechanism remains to be defined. In the present study, we determined the effects of bFGF on the regulation of cell growth as well as collagen and fibronectin expression in fibroblasts from normal human skin and from hypertrophic scars. We then explored the involvement of mitochondria in mediating bFGF-induced effects on the fibroblasts. We isolated and cultivated normal and hypertrophic scar fibroblasts from tissue biopsies of patients who underwent plastic surgery for repairing hypertrophic scars. The fibroblasts were then treated with different concentrations of bFGF (ranging from 0.1 to $1000 \mathrm{ng} / \mathrm{mL}$ ). The growth of hypertrophic scar fibroblasts became slower with selective inhibition of type I collagen production after exposure to bFGF. However, type III collagen expression was affected in both normal and hypertrophic scar fibroblasts. Moreover, fibronectin expression in the normal fibroblasts was up-regulated after bFGF treatment. bFGF $(1000 \mathrm{ng} / \mathrm{mL})$ also induced mitochondrial depolarization in hypertrophic scar fibroblasts $(P<0.01)$. The cellular ATP level decreased in hypertrophic scar fibroblasts $(P<0.05)$, while it increased in the normal fibroblasts following treatment with bFGF $(P<0.01)$. These data suggest that bFGF has differential effects and mechanisms on fibroblasts of the normal skin and hypertrophic scars, indicating that bFGF may play a role in the early phase of skin wound healing and post-burn scar formation.
\end{abstract}

Key words: bFGF; Hypertrophic scar; Fibroblast; Extracellular matrix; Mitochondria; Gene expression

\section{Introduction}

Wound healing is a dynamic and closely interactive process of various growth factors, fibroblasts, and the formation of new blood vessels and extracellular matrices. Molecularly, the growth factors or cytokines derived from monocyte/macrophage or lymphocytes are essential to attract different types of cells to move into the wound area. Fibroblasts play an important role in wound healing by producing a provisional wound healing matrix, including collagen and fibronectin (1). Some fibroblasts differentiate into myofibroblasts, which are principally responsible for tissue contraction, but also produce extracellular matrix components. In normal human wound healing, once tissue integrity has been restored, the myofibroblasts disappear from the scar (2). However, in pathological situations such as hypertrophic scar (HS), myofibroblasts persist in the tissue (3). In particular, increased activity of fibrogenic cytokines [e.g., transforming growth factor B1 (TGF-ß1), insulin-like growth factor 1 (IGF-1), and interleukin-1] and exaggerated responses to these cytokines may also play a role in post-burn scars such as fibrosis and keloids (3). The aberrant fibroblast phenotype also seems to contribute to the hypertrophic scars or post-burn scars, which may be due to the differential response of normal and HS fibroblasts to various growth factors. Specific growth factors targeted

Correspondence: Ke-Seng Zhao, Tonghe Rd, Southern Medical University, Guangzhou 510515, China. E-mail: zhaoks1937@yahoo.com

Received August 3, 2010. Accepted March 18, 2011. Available online April 1, 2011. Published May 16, 2011. 
would include TGF-ß1, IGF-1, or basic fibroblast growth factor (bFGF) $(4,5)$. It has been shown that bFGF is also a potent chemotactic factor for fibroblasts and endothelial cells, it can promote or inhibit cell differentiation, and it also functions as a potent angiogenic and neurotrophic factor $(6,7)$. Indeed, previous in vitro and in vivo studies have demonstrated that bFGF treatment effectively regulates granulation, tissue formation through apoptosis and inhibition of collagen synthesis and, therefore, can promote scarless repair $(8,9)$. The scar-inhibition effect of bFGF on sutured wounds was confirmed clinically, i.e., when bFGF was injected into the dermis or topically applied after surgery, no hypertrophic scars were formed later. In many cases, there was only a fine linear scar (10). However, the molecular mechanism underlying the action of bFGF in reducing the hypertrophic scar and enhancing wound healing remains to be demonstrated.

In the current study, we determined the role of bFGF during the regulation of type I and III collagen and fibronectin expression in fibroblasts derived from human normal skin and from hypertrophic scar biopsies. These proteins are structural molecules of the provisional matrix in wound repair. In addition, mitochondria may be an important mediator in extracellular matrix homeostasis and cell proliferation (11-13). Therefore, the mitochondrial membrane potential and cellular ATP level were measured before and after bFGF treatment of these fibroblasts. Our study provides evidence that mitochondria mediate the reversible regulation of bFGF capacity in wound healing and uncontrolled scar formation.

\section{Material and Methods}

\section{Cell cultures and treatments}

Fibroblasts were isolated from human skin biopsies obtained from five patients who underwent plastic surgery to correct excess scar tissue. The laboratory protocol was approved by the Medical and Ethics Committee of Southern Medical University and the People's Hospital of Guangdong Province and written informed consent was obtained from the patients. Fibroblasts were isolated from the dermis of the biopsies according to a method previously described $(14,15)$. The cells, at $70-80 \%$ confluence, were treated with different concentrations of bFGF in a serum-free medium.

\section{Transmission electron microscopy}

Ultrastructural analysis of fibroblasts was conducted by transmission electron microscopy. Cells were fixed for Epon-Araldite embedding in $2.5 \%$ glutaraldehyde in 0.1 $\mathrm{M}$ sodium cacodylate, $\mathrm{pH} 7.4$, and then post-fixed in $1 \%$

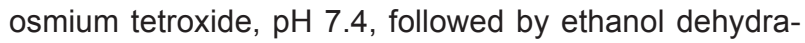
tion. Ultrathin sections were stained on-grid with $5 \%$ uranyl acetate and lead citrate, and examined with an electron microscope (Philip CM10, Holland).

\section{Measurement of fibroblast proliferation and death}

The fibroblasts were grown and treated or not with bFGF for $72 \mathrm{~h}$ before analysis. Cells were trypsinized with phosphate-buffered trypsin/EDTA at $37^{\circ} \mathrm{C}$ for $10 \mathrm{~min}$, and collected by centrifugation. The number of released cells was quantified by counting in a hemocytometer chamber, with viability assessed by $0.4 \%$ Trypan blue exclusion. The assay was repeated three times.

\section{Type I and III collagen and fibronectin assays}

Type I and III collagen and fibronectin levels in the conditioned culture media of human skin fibroblast cultures were measured using a commercially available ELISA kit according to manufacturer instructions (Adlitteram Diagnostic Laboratories Inc., China). The levels of type I and III collagen and fibronectin in the conditioned culture media were assayed in triplicate and measured with an automatic microplate reader (SpectraMax M5, USA) at a wavelength of $450 \mathrm{~nm}$.

\section{RNA isolation and reverse transcription polymerase chain reaction (RT-PCR)}

The fibroblasts were grown and treated with bFGF for 72 $\mathrm{h}$, and total RNA was extracted from the cells and converted into cDNA using an E.Z.N.A. total RNA kit (Omega Bio-tek, USA). The fibroblasts were further subjected to RT-PCR amplification of gene expression. APrimeScript ${ }^{\mathrm{TM}}$ RT-PCR kit (TaKaRa, Japan) was used according to manufacturer instructions (16). The following oligonucleotide primer sets (Invitrogen, USA) were designed and synthesized: type I collagen forward: 5'-CATGCCGTGACTTGAGACTCA-3' and reverse: 5'-AGGCGCATGAAGGCAAGTT-3', producing a 292-bp band; type III collagen forward: 5'-CGAGCTT CCCAGAACATCACA-3' and reverse: 5'-TTCGTGCAACC ATCCTCCA-3', producing a 170-bp band; fibronectin forward: 5'-TTCCTTGCTGGTATCATGGCA-3' and reverse: 5'-TATTCGGTTCCCGGTTCCA-3', producing a 156-bp band, and $\beta$-actin forward: 5'-GCCCTGAGGCACTCTT CCA-3' and reverse: 5'-GCGGATGTCCACGTCACA-3', producing a 101-bp band. The reaction mixtures were denatured at $95^{\circ} \mathrm{C}$ for $10 \mathrm{~min}$, and then amplified for 30 cycles at $94^{\circ} \mathrm{C}$ for $30 \mathrm{~s}, 55^{\circ} \mathrm{C}$ for $30 \mathrm{~s}$, and $72^{\circ} \mathrm{C}$ for $1 \mathrm{~min}$, with a final extension at $72^{\circ} \mathrm{C}$ for $10 \mathrm{~min}$ and later stored at $4^{\circ} \mathrm{C}$. The PCR products were then electrophoresed on a $2 \%$ agarose gel. The expression levels of type I and III collagen and fibronectin were quantified and normalized with $\beta$-actin using densitometry.

\section{Determination of mitochondrial membrane potential}

Mitochondrial membrane potential $\left(\Delta \Psi_{\mathrm{m}}\right)$ was measured using the fluorescent probe $\mathrm{JC}-1$. This dye normally exists in the mitochondrial matrix as a dimeric configuration emitting a red fluorescence, but then assumes a monomer configuration, emitting a green fluorescence when $\Delta \Psi_{\mathrm{m}}$ depolarizes. The fibroblasts were grown and treated with 
bFGF for $72 \mathrm{~h}$ and trypsinized and $10^{5}$ fibroblasts were collected by centrifugation (10 min, $112 \mathrm{~g}$ ). After washing twice with PBS, the cells were incubated with $2 \mu \mathrm{M} \mathrm{JC}-1$ dye for $15 \mathrm{~min}$ at $37^{\circ} \mathrm{C}$ in a humidified incubator. JC-1 was excited at $488 \mathrm{~nm}$ and the aggregate signal (red) was analyzed at $590 \mathrm{~nm}$ (FL2). The monomer signal (green) was analyzed at $525 \mathrm{~nm}$ (FL1) on a FACScan instrument (Becton Dickinson, USA) using the Cell Quest software (Becton Dickinson). The ratio of the mean red to the mean green $\mathrm{JC}-1$ fluorescence was calculated as a relative mitochondrial membrane potential as described in a previous study (17).

\section{Cytosolic ATP measurements}

Relative levels of cellular ATP were assayed using the CellTiter 96 assay kit from Promega (USA) according to manufacturer instructions (18). Briefly, $5 \times 10^{3}$ cells were plated onto a 96-well plate (BD Biosciences, USA) and grown for $72 \mathrm{~h}$. The cells were then mixed with $0.1 \mathrm{~mL}$ of the CellTiter substrate for $10 \mathrm{~min}$ and the luminescent output was read with an automatic microplate reader (SpectraMax M5). The cellular ATP correlations are reported as percent luminescence at each drug concentration in relation to the luminescence of untreated normal cells.

\section{Statistical analysis}

Data are reported as means \pm SEM and were analyzed by the Student paired $t$-test and one-way ANOVA using SPSS 13.0 for Windows. $\mathrm{P} \leq 0.05$ was considered to be statistically significant.

\section{Results}

\section{Ultrastructural analysis of the two types of fibroblasts from normal skin and hypertrophic scars}

To determine whether the fibroblasts isolated from HS were phenotypically distinct from normal human skin fibroblasts, we examined their ultrastructure by electron microscopy. Fibroblasts from HS have a myofibroblast-like structure with a fibronexus (Figure 1B), a subplasmalemmal complex involving intracellular actin microfilaments (Figure $1 \mathrm{C})$, and an external lamina. However, this structure was not observed in fibroblasts from normal human skin (Figure $1 \mathrm{~A}$ ). Moreover, more than $80 \%$ of the HS fibroblasts also showed myofibroblast differentiation under electron microscopy, i.e., numerous microfilament bundles parallel to the main axis of the cell, dense plaques at the plasma membrane, and/or the external lamina surrounding the cells (data not shown).

\section{Effect of bFGF on fibroblast proliferation and death}

To elucidate the effects of bFGF on the growth of the two types of fibroblasts, the fibroblasts were treated with or without bFGF, 0.1-1000 $\mathrm{ng} / \mathrm{mL}$, for $72 \mathrm{~h}$ and then counted. In the absence of bFGF, the mean number of HS fibroblasts was $19.43 \pm 1.27 \times 10^{5}$, and the number of normal fibroblasts was $9.57 \pm 0.78 \times 10^{5}(P<0.05)$. In the presence of $100 \mathrm{ng} / \mathrm{mL} \mathrm{bFGF}$, the mean number of normal fibroblasts
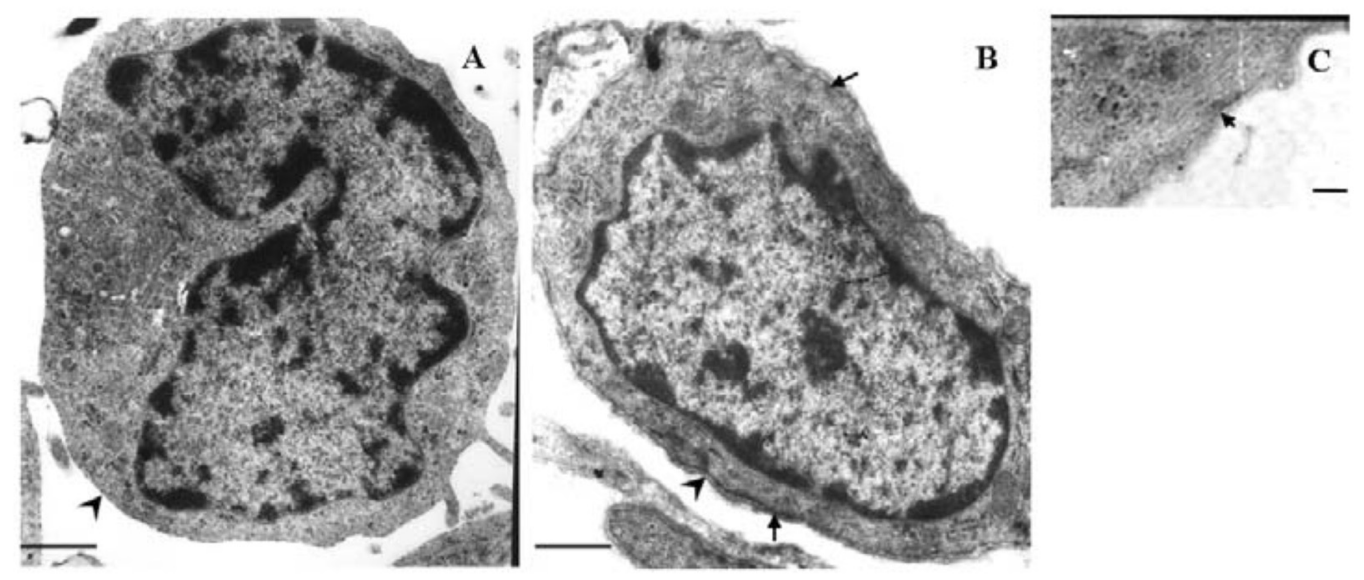

Figure 1. Ultrastructure of normal skin and hypertrophic scar (HS) fibroblasts. $A$, The normal fibroblast showed the absence of myofibroblast-like features (arrowhead). $B$, Transmission electron micrograph of a cross-section of HS fibroblasts showed fibroblast-to-myofibroblast transdifferentiation according to features that are similar to myofibroblasts, with the external lamina (arrowhead) and with hemidesmosome-like structure corresponding to the so-called fibronexus (arrows). C, The characteristics of actin microfilament bundles and the subplasmalemmal dense plaque (arrow) in another transformed HS fibroblast. Bars: A and B $=1 \mu \mathrm{m} ; \mathrm{C}=0.5 \mu \mathrm{m}$. 
increased to a peak of $58.17 \pm 3.75 \times 10^{5}$ ( $\left.P<0.01\right)$. In the presence of $100 \mathrm{ng} / \mathrm{mL} b F G F$, the mean number of HS fibroblasts increased to a peak of $41.50 \pm 5.67 \times 10^{5}(\mathrm{P}$ $<0.01$ ). However, in the presence of $1000 \mathrm{ng} / \mathrm{mL} b F G F$, the mean number of $\mathrm{HS}$ fibroblasts was reduced to 10.20 $\pm 0.79 \times 10^{5}(\mathrm{P}<0.01)$, while that of normal fibroblasts was $30.13 \pm 3.90 \times 10^{5}(\mathrm{P}<0.01$; Figure $2 \mathrm{~A})$. Therefore, $1000 \mathrm{ng} / \mathrm{mL}$ bFGF reduced HS fibroblast proliferation by approximately $48 \%$. Furthermore, the number of Trypan blue-positive dead cells increased significantly in cultures treated with $1000 \mathrm{ng} / \mathrm{mL}$ bFGF for $72 \mathrm{~h}(\mathrm{P}<0.01$; Figure $2 \mathrm{C}$ ). However, there was no significant difference in the number of dead cells in the normal fibroblast group (Figure 2B).

\section{Effect of bFGF on fibroblast collagen production}

We determined the effect of bFGF on the regulation of collagen expression in both normal and HS fibroblasts. Type I collagen produced by HS fibroblasts was $0.031 \pm$ $0.004 \mu \mathrm{g} \cdot \mathrm{mL}^{-1} \cdot \mathrm{mg}$ protein ${ }^{-1}$, which was significantly higher than that produced by normal fibroblasts $(0.017 \pm 0.004$ $\mu \mathrm{g} \cdot \mathrm{mL}^{-1} \cdot \mathrm{mg}$ protein $\left.{ }^{-1}\right)$ in the absence of bFGF culture $(P$ $<0.05)$. However, in the presence of 1, 10, 100, and 1000 $\mathrm{ng} / \mathrm{mL}$ bFGF, type I collagen in HS fibroblasts significantly decreased to $0.015 \pm 0.008,0.014 \pm 0.006,0.016 \pm 0.005$, and $0.015 \pm 0.005 \mu \mathrm{g} / \mathrm{mL}$, respectively $(P<0.01)$. In contrast, after exposure to different concentrations of bFGF, the levels of expression of type I collagen were not significantly changed in normal fibroblasts (Figure 3). Expression of type III collagen was not significantly altered by bFGF treatment in either normal or HS fibroblasts (Figure 3).

\section{Effect of bFGF on fibroblast collagen mRNA expression}

Next, we also determined the effect of bFGF on the expression of collagen mRNA levels in these fibroblasts before and after bFGF treatment by semi-quantitative RT-PCR. As shown in Figure 4, the expression of type I collagen mRNA in HS fibroblasts was significantly higher than that in the normal fibroblasts in the absence of bFGF $(P<0.05)$. However, in the presence of $1,10,100$, and $1000 \mathrm{ng} / \mathrm{mL}$ bFGF, the expression of type I collagen mRNA significantly decreased to $62,60,61$, and $69 \%$ of the pretreated value, respectively $(P<0.01)$. In contrast, no significant changes were observed in normal fibroblasts after treatment with bFGF (Figure 4). In addition, the expression of type III collagen mRNA was not significantly changed after bFGF treatment (Figure 4).

\section{Effect of bFGF on fibroblast fibronectin expression}

As shown in Figures 3 and 4, the effects of bFGF on fibronectin $\mathrm{mRNA}$ and protein levels were also assessed in normal and HS fibroblasts. In the presence of bFGF, normal fibroblasts showed an increase in the expression of fibronectin protein $(P<0.01$; Figure 3$)$ and $\operatorname{mRNA}(P<$
0.01 ; Figure 4), whereas there was no significant difference in fibronectin expression in HS fibroblasts (Figures
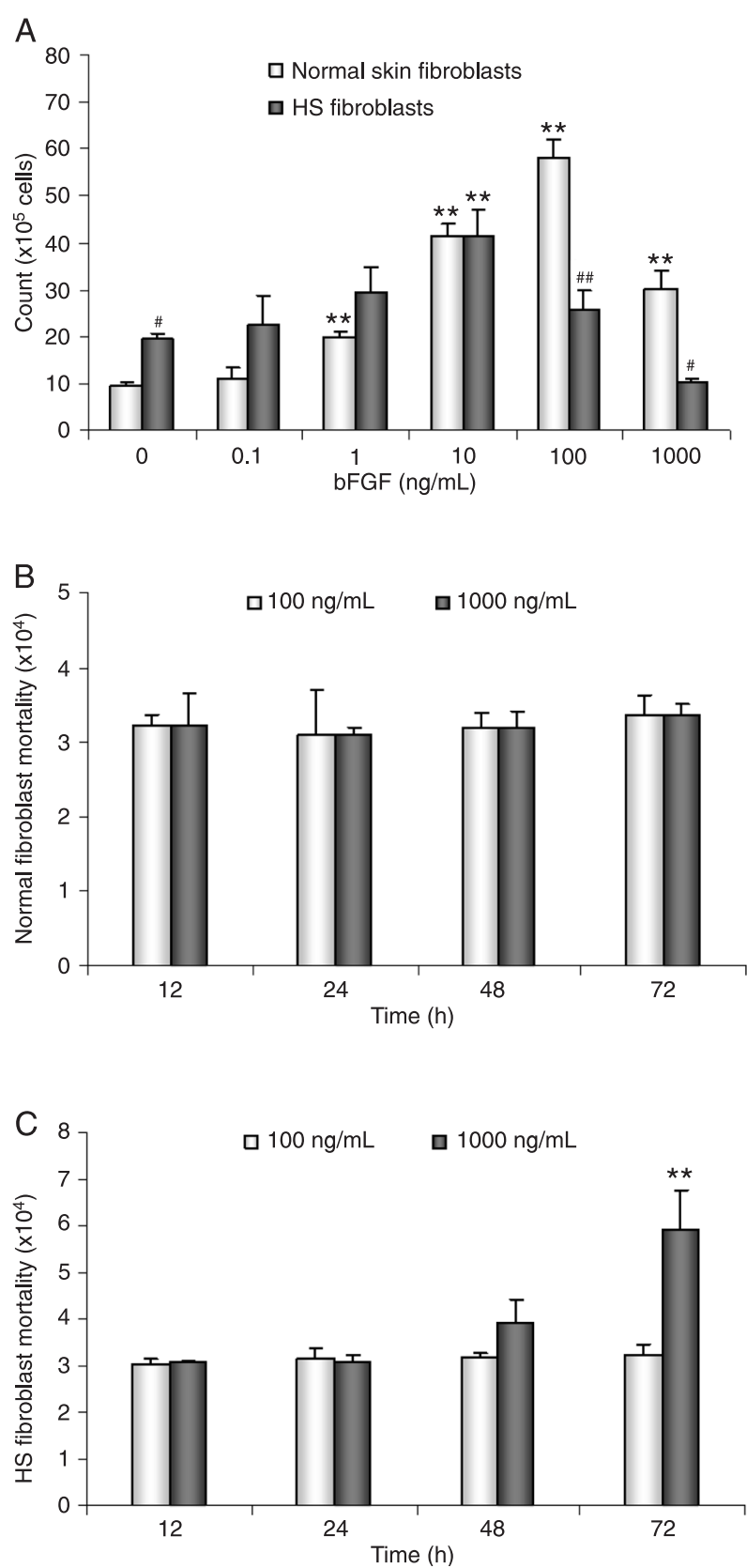

Figure 2. Effect of basic fibroblast growth factor (bFGF) on proliferation and death of normal skin and hypertrophic scar (HS) fibroblasts. $A$, The proliferation of HS fibroblasts was reduced compared to that of normal fibroblasts following treatment with $\mathrm{bFGF}$ at concentrations of 0.1 to $1000 \mathrm{ng} / \mathrm{mL}$ for $72 \mathrm{~h}$. B, bFGF treatment did not affect the mortality of normal fibroblasts. $C$, bFGF treatment resulted in an increase of HS fibroblast mortality. Data are reported as means \pm SEM. The experiments were performed in triplicate and repeated at least once. ${ }^{* *} \mathrm{P}<0.01 \mathrm{vs}$ untreated fibroblasts; $P<<0.05,{ }^{\#} P<0.01$ vs normal skin fibroblasts (paired $t$-test and one-way randomized ANOVA). 
3 and 4). The comparison of the generalized estimating equations between the two types of fibroblasts showed a statistically significant difference in fibronectin expression $(P<0.05)$.

\section{Effect of bFGF on the mitochondrial membrane potential and cellular ATP levels}

Previous studies have demonstrated that mitochondrial function plays an important role in the function and survival
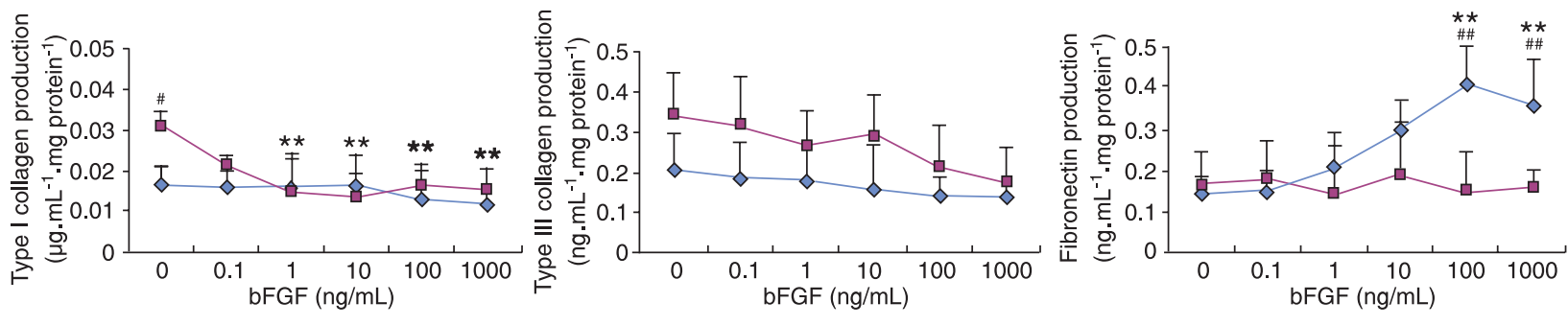

$\diamond$ Normal skin fibroblasts

a HS fibroblasts

Figure 3. Effect of basic fibroblast growth factor (bFGF) on type I/III collagen and fibronectin production by normal and hypertrophic scar (HS) fibroblasts. The cells were grown and treated with bFGF at concentrations of 0.1 to $1000 \mathrm{ng} / \mathrm{mL}$ for $72 \mathrm{~h}$ and subjected to ELISA. bFGF decreased type I collagen production by HS fibroblasts and increased fibronectin production by normal fibroblasts. Data are reported as means \pm SEM. The experiments were carried out in triplicate and repeated at least once. ${ }^{* *} \mathrm{P}<0.01 \mathrm{vs}$ untreated fibroblasts; ${ }^{\mathrm{P}}<0.05,{ }^{\#} \mathrm{P}<0.01$ vs normal skin fibroblasts (paired $t$-test and one-way randomized ANOVA).

A

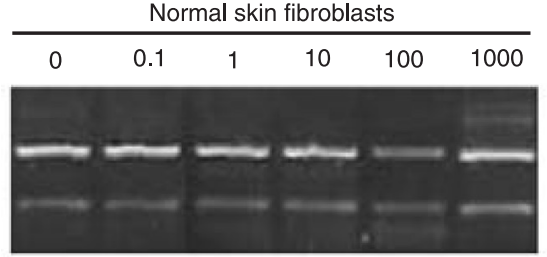

B

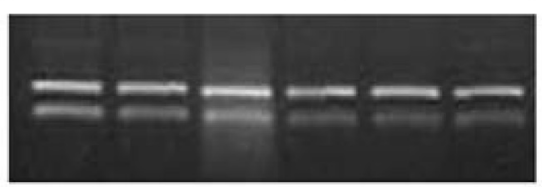

C

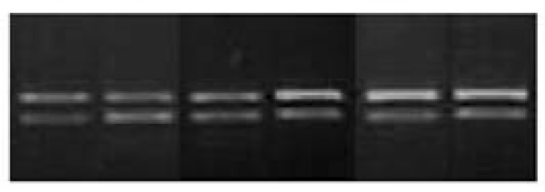

HS fibroblasts
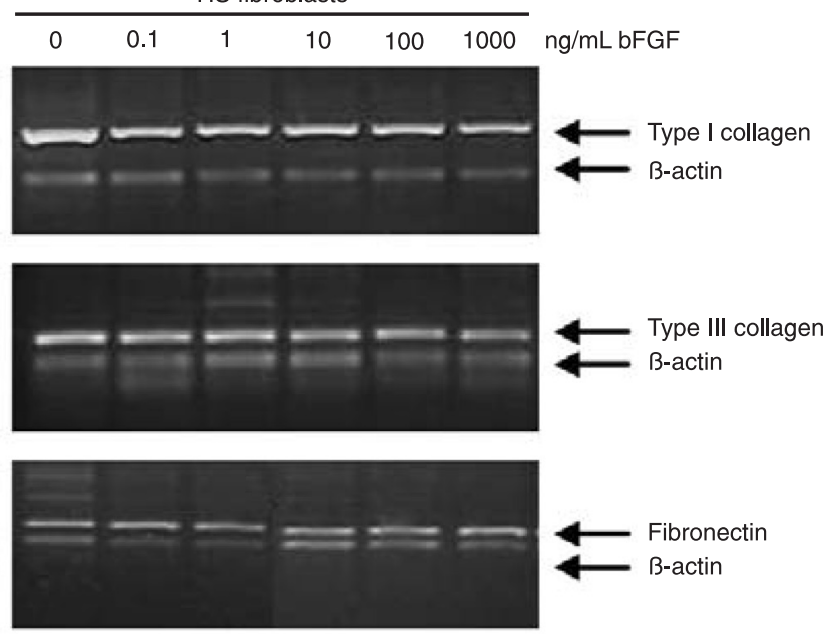
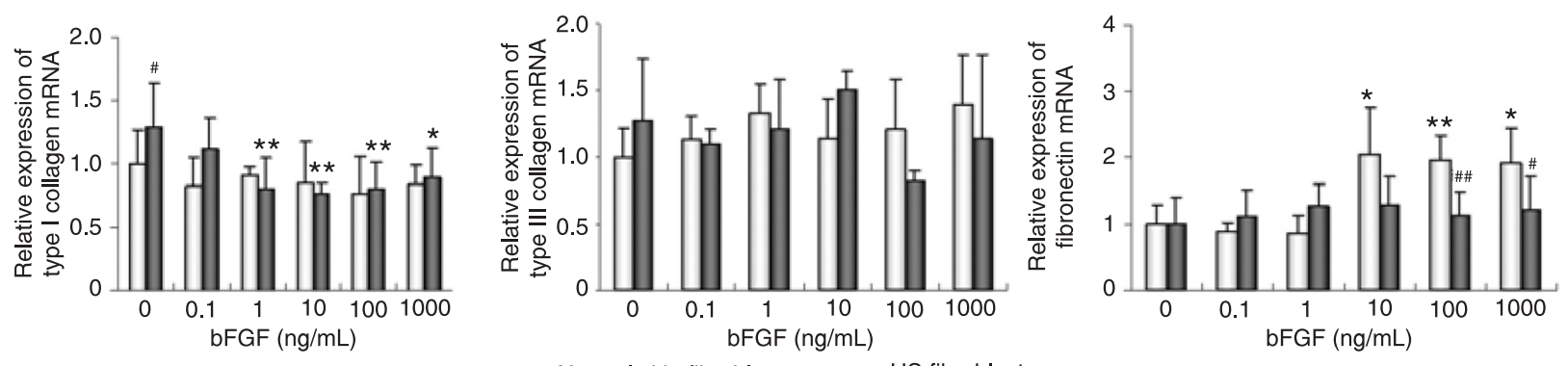

- Normal skin fibroblasts

- HS fibroblasts

Figure 4. Effect of basic fibroblast growth factor (bFGF) on the expression of type I/III collagen and fibronectin mRNA in normal and hypertrophic scar (HS) fibroblasts. The cells were grown and treated with bFGF at concentrations of 0.1 to $1000 \mathrm{ng} / \mathrm{mL}$ for $72 \mathrm{~h}$ and then subjected to RT-PCR analysis of type I (A), type III collagen (B), and fibronectin (C) expression. bFGF down-regulated type I collagen mRNA in HS fibroblasts and up-regulated fibronectin mRNA in normal fibroblasts. Data were normalized to a $\beta$-actin loading control. The bottom graphs showed the quantitative RT-PCR data. Data are reported as means \pm SEM. ${ }^{*} \mathrm{P}<0.05,{ }^{* *} \mathrm{P}<0.01$ vs untreated fibroblasts; ${ }^{\mathrm{P}}<0.05,{ }^{\# \mathrm{P}}<0.01$ vs normal skin fibroblasts (paired $t$-test and one-way randomized ANOVA). 

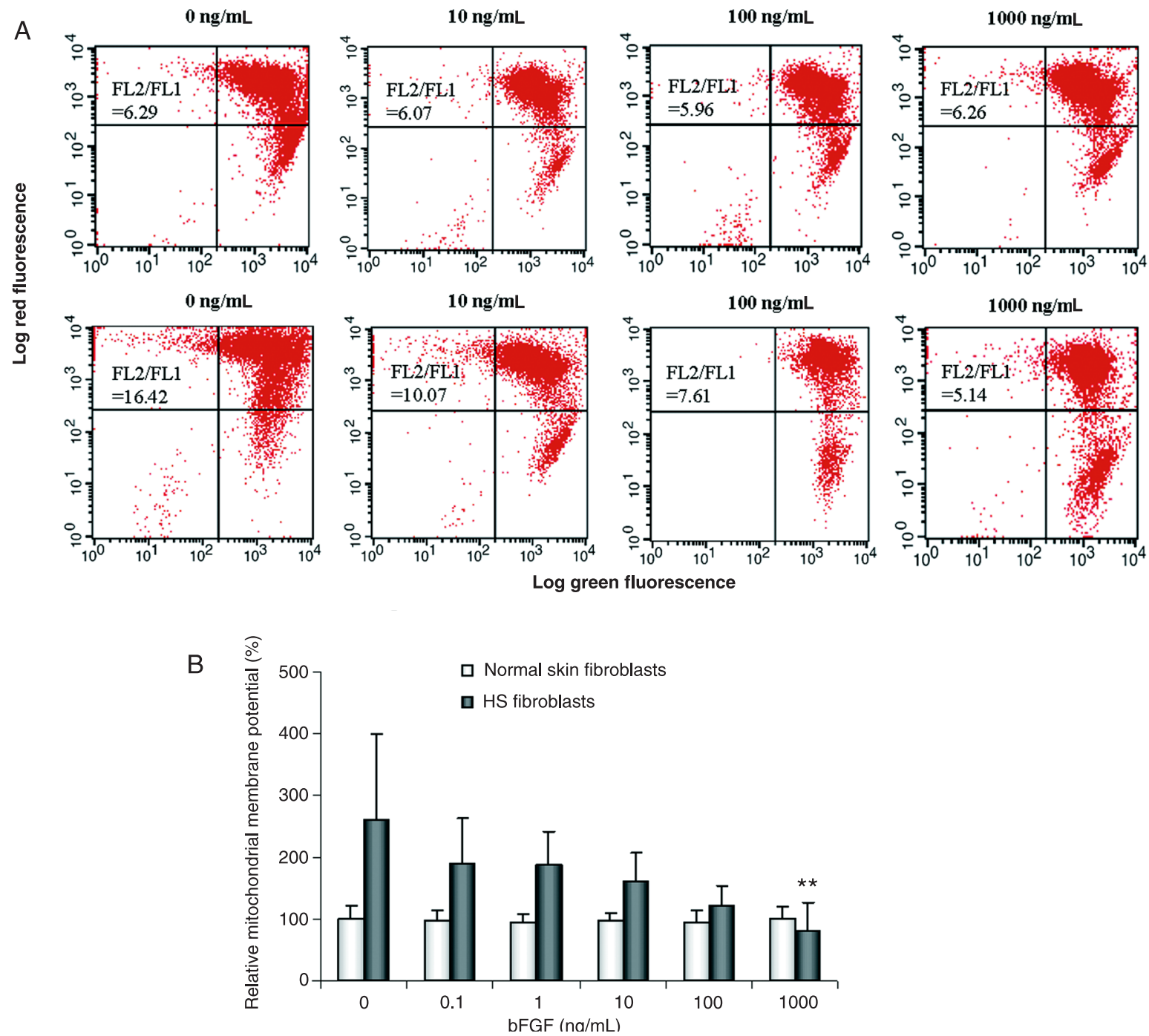

Figure 5. Flow cytometry analysis of the mitochondrial membrane potential in normal and hypertrophic scar (HS) fibroblasts. Cells were grown and treated with basic fibroblast growth factor (bFGF) at concentrations of 0.1 to $1000 \mathrm{ng} / \mathrm{mL}$ for $72 \mathrm{~h}$ and then subjected to JC-1 staining and flow cytometry. The relative mitochondrial membrane potential was expressed as the ratio of $\mathrm{JC}-1$ red fluorescence (upper quadrant) to green fluorescence (lower right quadrant) in the normal (A, top) and HS fibroblasts (A, bottom). bFGF depolarized HS fibroblasts mitochondria, with no effect on mitochondrial membrane potential in normal fibroblasts. The quantitative data $(B)$ are reported as means \pm SEM. ${ }^{* *} \mathrm{P}<0.01 \mathrm{vs}$ untreated fibroblasts (paired $t$-test and one-way randomized ANOVA).

of fibroblasts for cell repair, growth, and extracellular matrix production during wound healing $(19,20)$. We, therefore, examined the changes in mitochondrial function after treating the two types of fibroblasts with bFGF by detecting changes in mitochondrial membrane potential and in cellular ATP levels. We observed that changes in the ratio of $\mathrm{JC}-1$ red to green fluorescence in $\mathrm{HS}$ fibroblasts decreased after bFGF treatment (Figure 5A). The relative mitochondrial membrane potential of HS fibroblasts significantly decreased from a pretreatment level of $261.14 \pm 138.29$ to $81.68 \pm 44.80 \%$ after treatment with $1000 \mathrm{ng} / \mathrm{mL}$ bFGF $(P<0.01$; Figure
$5 B)$, suggesting that the mitochondrial depolarization of $H S$ fibroblasts was induced by bFGF. bFGF treatment $(10,100$ and $1000 \mathrm{ng} / \mathrm{mL}$ ) also induced a significant increase in cellular ATP levels in normal fibroblasts $(P<0.01)$. However, in the presence of $1000 \mathrm{ng} / \mathrm{mL} \mathrm{bFGF}$, intracellular ATP levels were $38.16 \%$ lower than the pretreated level in HS fibroblasts $(P<0.05$; Figure 6$)$.

\section{Discussion}

The process of fibrosis and normal wound healing is 
closely associated with fibroblast growth and collagen formation. Excessive myofibroblast differentiation and extracellular matrix formation may be involved in hypertrophic scarring and formation of post-burn scars, such as fibrosis and keloids (3), resulting in hypertrophic scars, the management of which remains a challenge in clinical practice. However, there may be phenotypic differences between fibroblasts from normal skin and from hypertrophic scars in response to cytokines. bFGF, a self-limited and potent wound-healing agent, is being widely used for research in this field $(9,10,21)$, although the underlying molecular mechanism remains to be determined. It has been reported that mitochondria are related to myofibroblast differentiation (22). In the present study, we determined differential regulation of growth and collagen and fibronectin synthesis as well as mitochondrial membrane potential in normal and HS fibroblasts after treatment with bFGF.

We observed that production of type I collagen by HS fibroblasts was much higher than production by normal skin fibroblasts in the absence of bFGF, while production of type I collagen by HS fibroblasts was significantly inhibited by a high dose of bFGF. A previous study revealed that excessive production and deposition of type I collagen played an important role in hypertrophic scar formation (23). In this respect, a previous study showed that bFGF was able to reduce type I collagen levels in keloid fibroblasts as detected by immunostaining (8). The reduction of type I collagen production was also observed under immunofluorescent and scanning electron microscopy after administration of bFGF for 6 weeks (24). The inhibition was associated with transcriptional down-regulation of type I collagen gene expression $(8,25)$. Our data are in agreement with these previous data. Furthermore, although the rate of type III collagen synthesis is increased in the early wound healing period $(26,27)$, our data showed that this type of collagen might not be involved in bFGF-mediated wound healing. Fibronectin is also highly up-regulated after wound healing and acts as a provisional matrix, promoting fibroblast migration in the early phase of wound healing $(28,29)$. Excessive deposition of fibronectin may contribute, at least in part, to hypertrophic scar formation (30). The present experiments indicated that bFGF increased fibronectin production, possibly by the up-regulation of fibronectin gene expression in normal fibroblasts, but this did not occur in HS fibroblasts. Therefore, these data suggest that bFGF-mediated production of extracellular matrix and gene expression were cell phenotype-dependent. In addition, identification of bFGF in the inhibition of type I collagen expression in HS fibroblasts and the increase of fibronectin in normal fibroblasts provide insightful information regarding the potential mechanism of action of bFGF in the early phase of wound healing and in post-burn scar formation.

Previously reported in vivo data showed that the early subcutaneous administration of bFGF inhibited the differentiation of fibroblasts and excessive formation of myofi-

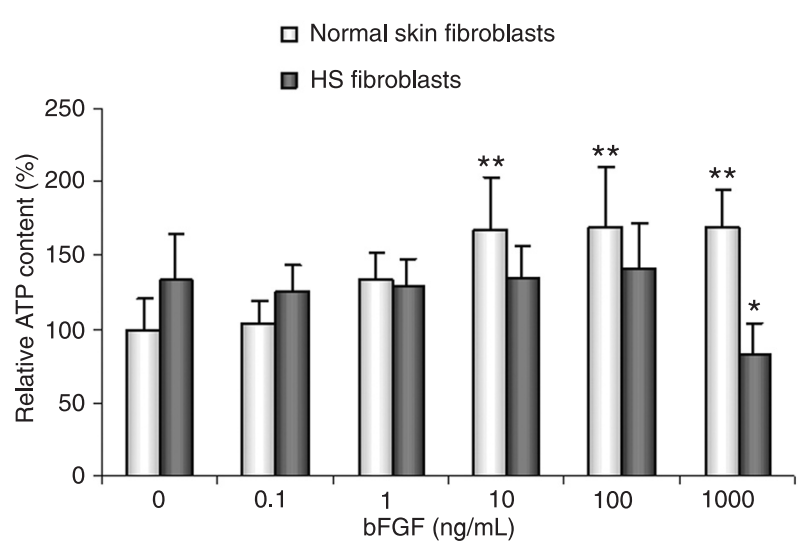

Figure 6. Effect of basic fibroblast growth factor (bFGF) on the regulation of cellular ATP levels in normal and hypertrophic scar (HS) fibroblasts. Cells were grown and treated with bFGF at concentrations of 0.1 to $1000 \mathrm{ng} / \mathrm{mL}$ for $72 \mathrm{~h}$ and then subjected to the luciferin-luciferase assay. bFGF decreased the intracellular ATP levels in HS fibroblasts and increased the ATP levels in the normal fibroblasts. Data are reported as means \pm SEM. ${ }^{*} P<$ $0.05,{ }^{* *} \mathrm{P}<0.01$ vs untreated fibroblasts (paired $t$-test and oneway randomized ANOVA).

broblasts in wound healing (8). It was recently reported that bFGF-induced granulation tissue formation was due to the increase in cell proliferation that exceeded cell apoptosis on days 4 and 7; however, suppression of granulation tissue formation was mainly due to the increased fibroblast apoptosis on day 14 (10). The present data suggest that growth of HS fibroblasts became slower than that of normal fibroblasts after exposure to bFGF. Taken together, these data suggest an explanation for the difference in response to bFGF by normal fibroblasts and HS fibroblasts.

Mitochondria are perceived to be necessary for controlling cell growth, differentiation, death, gene expression and other key cellular processes (31). Wound healing is a process that uses cell energy to move the wound cells and to form the extracellular matrix. For example, fibroblasts can survive but not replicate or synthesize collagen in a low energy environment (32). It is evident that mitochondrial functioning varies with the changes in the extracellular matrix composition to regulate the extracellular matrix. Transforming growth factor- $\beta 1$ has been recently demonstrated to be able to modify extracellular matrix composition and structure and to affect mitochondrial function (20). As a result, knowledge of the differences in mitochondria between normal fibroblasts and HS fibroblasts treated with bFGF would be important in determining how bFGF regulates the scarless wound repair process. However, there are no previous data documenting the differences in mitochondrial functions between normal fibroblasts and HS fibroblasts after treatment with bFGF. In the present study, it was observed that bFGF had no effect on the modulation of the $\Delta \Psi$ m in normal skin fibroblasts, but led to depolarized mitochondria in HS 
fibroblasts. Maintaining mitochondrial membrane potential was found to be a prominent mechanism in the production of cellular ATP by means of the mitochondria $(33,34)$. Consistent with this mechanism, our results showed that bFGF induced an increase of relative ATP levels in normal fibroblasts and a decrease in cellular ATP in HS fibroblasts, which might contribute to the different growth rate between normal fibroblasts and HS fibroblasts. Interestingly, a high concentration of bFGF ( $1000 \mathrm{ng} / \mathrm{mL}$ in the current study) led to significant dissipation of $\Delta \Psi \mathrm{m}$ and $\mathrm{HS}$ fibroblast death, but did not affect normal fibroblasts. It has been previously demonstrated that mitochondrial permeability transition causes $\Delta \Psi_{\mathrm{m}}$ dissipation, uncoupling of oxidative phosphorylation, ATP depletion, and equilibration of small solutes and ions between the cytosol and the mitochondrial matrix to control cell death or survival (31). However, this matter needs to

\section{References}

1. Werner S, Grose R. Regulation of wound healing by growth factors and cytokines. Physiol Rev 2003; 83: 835-870.

2. Singer AJ, Clark RA. Cutaneous wound healing. $N$ Engl $J$ Med 1999; 341: 738-746.

3. Wynn TA. Cellular and molecular mechanisms of fibrosis. $J$ Pathol 2008; 214: 199-210.

4. Tiede S, Ernst N, Bayat A, Paus R, Tronnier V, Zechel C. Basic fibroblast growth factor: a potential new therapeutic tool for the treatment of hypertrophic and keloid scars. Ann Anat 2009; 191: 33-44.

5. Zhang K, Garner W, Cohen L, Rodriguez J, Phan S. Increased types I and III collagen and transforming growth factor-beta $1 \mathrm{mRNA}$ and protein in hypertrophic burn scar. $J$ Invest Dermatol 1995; 104: 750-754.

6. Ortega S, Ittmann M, Tsang SH, Ehrlich M, Basilico C. Neuronal defects and delayed wound healing in mice lacking fibroblast growth factor 2. Proc Natl Acad Sci U S A 1998; 95: 5672-5677.

7. Abraham JA, Klagsbrun M. Modulation of wound repair by members of the fibroblast growth factor family. In: Clark RAF (Editor), The molecular and cellular biology of wound repair. 2nd edn. New York: Plenum Press; 1996. p 195-248.

8. Tan EM, Rouda S, Greenbaum SS, Moore JH Jr, Fox JW, Sollberg S. Acidic and basic fibroblast growth factors downregulate collagen gene expression in keloid fibroblasts. $\mathrm{Am}$ J Pathol 1993; 142: 463-470.

9. Akasaka Y, Ono I, Yamashita T, Jimbow K, Ishii T. Basic fibroblast growth factor promotes apoptosis and suppresses granulation tissue formation in acute incisional wounds. $J$ Pathol 2004; 203: 710-720.

10. Ono I, Akasaka Y, Kikuchi R, Sakemoto A, Kamiya T, Yamashita $\mathrm{T}$, et al. Basic fibroblast growth factor reduces scar formation in acute incisional wounds. Wound Repair Regen 2007; 15: 617-623.

11. Irwin WA, Bergamin N, Sabatelli P, Reggiani C, Megighian A, Merlini L, et al. Mitochondrial dysfunction and apoptosis in myopathic mice with collagen VI deficiency. Nat Genet 2003; 35: 367-371.

12. Wu D, Chen X, Guo D, Hong Q, Fu B, Ding R, et al. Knock- be further investigated regarding whether bFGF-induced death of HS fibroblasts is due to changes in mitochondrial function or due to bFGF-induced myofibroblast death for eliminating this kind of myofibroblast-differentiated fibroblast in pathological tissue contractures. Data from the current study suggest that the effect of bFGF on skin fibroblasts may occur through changes in mitochondrial function, indicating that future studies could target bFGF for the control of wound healing and for antifibrosis therapy.

\section{Acknowledgments}

Research supported in part by the National Natural Science Foundation of China grant \#30672179 and the Guangdong Province Academic Fund (\#04000570). down of fibronectin induces mitochondria-dependent apoptosis in rat mesangial cells. J Am Soc Nephrol 2005; 16 : 646-657.

13. Hoye AT, Davoren JE, Wipf P, Fink MP, Kagan VE. Targeting mitochondria. Acc Chem Res 2008; 41: 87-97.

14. Nakano T, Scott PG. Purification and characterization of a gelatinase produced by fibroblasts from human gingiva. Biochem Cell Biol 1986; 64: 387-393.

15. Hanasono MM, Lum J, Carroll LA, Mikulec AA, Koch RJ. The effect of silicone gel on basic fibroblast growth factor levels in fibroblast cell culture. Arch Facial Plast Surg 2004; 6: 88-93.

16. Ufer C, Wang CC, Fahling M, Schiebel H, Thiele BJ, Billett $\mathrm{EE}$, et al. Translational regulation of glutathione peroxidase 4 expression through guanine-rich sequence-binding factor 1 is essential for embryonic brain development. Genes Dev 2008; 22: 1838-1850.

17. Kirshner JR, He S, Balasubramanyam V, Kepros J, Yang CY, Zhang $\mathrm{M}$, et al. Elesclomol induces cancer cell apoptosis through oxidative stress. Mol Cancer Ther 2008; 7: 23192327.

18. Pfister O, Oikonomopoulos A, Sereti KI, Sohn RL, Cullen D, Fine GC, et al. Role of the ATP-binding cassette transporter Abcg2 in the phenotype and function of cardiac side population cells. Circ Res 2008; 103: 825-835.

19. Gordon HM, Kucera G, Salvo R, Boss JM. Tumor necrosis factor induces genes involved in inflammation, cellular and tissue repair, and metabolism in murine fibroblasts. $\mathrm{J} \mathrm{Im}$ munol 1992; 148: 4021-4027.

20. de Cavanagh EM, Ferder M, Inserra F, Ferder L. Angiotensin II, mitochondria, cytoskeletal, and extracellular matrix connections: an integrating viewpoint. Am J Physiol Heart Circ Physiol 2009; 296: H550-H558.

21. Pierce GF, Tarpley JE, Yanagihara D, Mustoe TA, Fox GM, Thomason A. Platelet-derived growth factor (BB homodimer), transforming growth factor-beta 1 , and basic fibroblast growth factor in dermal wound healing. Neovessel and matrix formation and cessation of repair. Am J Pathol 1992; 140: $1375-1388$. 
22. Novo E, Marra F, Zamara E, Valfre di Bonzo L, Caligiuri A, Cannito S, et al. Dose dependent and divergent effects of superoxide anion on cell death, proliferation, and migration of activated human hepatic stellate cells. Gut 2006; 55: 9097.

23. Prockop DJ, Kivirikko KI. Collagens: molecular biology, diseases, and potentials for therapy. Annu Rev Biochem 1995; 64: 403-434.

24. Choi W, Kawanabe H, Sawa $Y$, Taniguchi K, Ishikawa $H$. Effects of bFGF on suppression of collagen type I accumulation and scar tissue formation during wound healing after mucoperiosteal denudation of rat palate. Acta Odontol Scand 2008; 66: 31-37.

25. Trojanowska M, LeRoy EC, Eckes B, Krieg T. Pathogenesis of fibrosis: type 1 collagen and the skin. J Mol Med 1998; 76: 266-274.

26. Stenfeldt K, Johansson C, Hellstrom S. The collagen structure of the tympanic membrane: collagen types I, II, and III in the healthy tympanic membrane, during healing of a perforation, and during infection. Arch Otolaryngol Head Neck Surg 2006; 132: 293-298.

27. Boudko SP, Engel J, Okuyama K, Mizuno K, Bachinger HP, Schumacher MA. Crystal structure of human type III collagen Gly991-Gly1032 cystine knot-containing peptide shows both $7 / 2$ and 10/3 triple helical symmetries. J Biol Chem
2008; 283: 32580-32589.

28. Knox P, Crooks S, Rimmer CS. Role of fibronectin in the migration of fibroblasts into plasma clots. J Cell Biol 1986; 102: 2318-2323.

29. Kwon AH, Qiu Z, Hirao Y. Topical application of plasma fibronectin in full-thickness skin wound healing in rats. Exp Biol Med 2007; 232: 935-941.

30. Sible JC, Eriksson E, Smith SP, Smith N. Fibronectin gene expression differs in normal and abnormal human wound healing. Wound Repair Regen 1994; 2: 3-19.

31. Kroemer G, Galluzzi L, Brenner C. Mitochondrial membrane permeabilization in cell death. Physiol Rev 2007; 87: 99163.

32. Hunt TK, Pai MP. The effect of varying ambient oxygen tensions on wound metabolism and collagen synthesis. Surg Gynecol Obstet 1972; 135: 561-567.

33. Chalmers S, McCarron JG. The mitochondrial membrane potential and $\mathrm{Ca}^{2+}$ oscillations in smooth muscle. J Cell Sci 2008; 121: 75-85.

34. Serrano MC, Pagani R, Manzano M, Comas JV, Portoles MT. Mitochondrial membrane potential and reactive oxygen species content of endothelial and smooth muscle cells cultured on poly(epsilon-caprolactone) films. Biomaterials 2006; 27 : $4706-4714$ 